\title{
USO DE INDICADORES NA AVALIAÇÃO DO \\ SERVIÇO DE EDUCAÇÃO PERMANENTE: \\ REFLEXÃO DOS PILARES DA QUALIDADE
}

\author{
USE OF INDICATORS IN THE EVALUATION OF THE \\ PERMANENT EDUCATION SERVICE: REFLECTION OF \\ THE QUALITY PILLARS
}

\section{USO DE INDICADORES EN LA EVALUACIÓN DEL SERVICIO DE EDUCACIÓN PERMANENTE: REFLEXIÓN DE LOS PILARES DE LA CALIDAD}

\author{
Graziele Ribeiro Bitencourt ${ }^{1}$ \\ Andreia Fabia de Melo Ferreira ${ }^{2}$ \\ Maria Helena de Souza Praça do Amaral ${ }^{3}$ \\ Stella Maris Gomes Renault ${ }^{4}$ \\ Jaqueline Olímpio da Silva ${ }^{5}$ \\ Katerine Moraes dos Santos ${ }^{6}$
}

Como citar este artigo: Bitencourt GR, Ferreira AFM, Amaral MHSP, Renault SMG, Silva JO, Santos KM. Uso de indicadores na avaliação do serviço de educação permanente: reflexão dos pilares da qualidade. Rev baiana enferm. 2021;35:e36844.

Objetivo: refletir sobre o uso de indicadores na avaliação da qualidade do serviço de educação permanente. Método: estudo descritivo, tipo análise reflexiva da literatura, à luz dos pilares da qualidade. Resultados: a avaliação da qualidade do serviço de educação permanente remete ao monitoramento dos indicadores associados à frequência dos profissionais nas atividades propostas, abandono na sequência dos treinamentos e satisfação com as atividades. Outros indicadores são relacionados ao próprio serviço de educação permanente, mediante o monitoramento da taxa de cancelamento das ações, tempo médio de capacitações e o valor investido no planejamento das ações. Dessa forma, a avaliação dos indicadores com base nos pilares da qualidade de eficácia, efetividade, eficiência, aceitabilidade, legitimidade e equidade pode auxiliar no planejamento e na análise dos treinamentos ofertados. Conclusão: o uso de indicadores na educação permanente favorece a análise retrospectiva e prospectiva das atividades por meio dos próprios profissionais e do serviço de educação permanente.

Descritores: Educação Permanente. Processo de Trabalho. Serviços de Saúde. Indicadores de Serviços. Gestão em Saúde.

Objective: to reflect on the use of indicators in the evaluation of the quality of the continuing education service. Method: descriptive study, reflective analysis of literature, in the light of the quality pillars. Results: the evaluation of

\footnotetext{
Enfermeira. Doutora em Enfermagem. Professora Assistente da Universidade Federal do Rio de Janeiro. Rio de Janeiro, Brasil. gra_uff@yahoo.com.br. http://orcid. org/0000-0002-9130-9307.

Enfermeira. Pesquisadora Independente. Rio de Janeiro, Rio de Janeiro, Brasil. http://orcid.org/0000-0002-9952-7|9X.

Enfermeira. Pesquisadora Independente. Rio de Janeiro, Rio de Janeiro, Brasil. http://orcid.org/0000-0002-7| 87-9206.

Enfermeira e Obstetra. Mestrado Profissional em Saúde e Tecnologia no Espaço Hospitalar. Pesquisadora Independente. Rio de Janeiro, Rio de Janeiro, Brasil. http:// orcid.org/0000-0002-6908-3364.

Pedagoga. Pesquisadora Independente. Rio de Janeiro, Rio de Janeiro, Brasil. http://orcid.org/0000-0003-3327-2I60.

Enfermeira. Mestre em Enfermagem. Enfermeira assistencial do Hospital Universitário Gaffrée e Guinle, da Universidade Federal do Estado do Rio de Janeiro. Rio de Janeiro, Rio de Janeiro, Brasil. http://orcid.org/0000-0002-2064-5207.
} 
the quality of the permanent education service refers to the monitoring of indicators associated with the attendance of professionals in the proposed activities, abandonment in following training and satisfaction with activities. Other indicators are related to the permanent education service itself, through the monitoring of the cancellation rate of actions, average training time and the value invested in the planning of actions. Thus, the evaluation of the indicators based on the pillars of quality of efficacy, effectiveness, efficiency, acceptability, legitimacy and equity is able to assist in the planning and analysis of the training offered. Conclusion: the use of indicators in permanent education favors the retrospective and prospective analysis of the activities by the professionals themselves and by the permanent education service.

Descriptors: Permanent Education. Work Process. Health Services. Services Indicators. Health Management.

Objetivo: reflexionar sobre el uso de indicadores en la evaluación de la calidad del servicio de educación permanente. Método: estudio descriptivo, análisis reflexivo de la literatura, a la luz de los pilares de la calidad. Resultados: la evaluación de la calidad del servicio de educación permanente se refiere al monitoreo de los indicadores asociados a la frecuentación de los profesionales en las actividades propuestas, el abandono en la secuencia de la formación y la satisfacción con las actividades. Otros indicadores se relacionan con el propio servicio de educación permanente, a través del monitoreo de la tasa de cancelación de acciones, el tiempo promedio de capacitación y el valor invertido en la planificación de acciones. Así pues, la evaluación de los indicadores basados en los pilares de la calidad de la eficacia, efectividad, eficiencia, aceptabilidad, legitimidad y equidad puede auxiliar en la planificación y el análisis de la formación ofrecida. Conclusión: la utilización de indicadores en la educación permanente favorece el análisis retrospectivo y prospectivo de las actividades a través de los propios profesionales y del servicio de educación permanente.

Descriptores: Educación Permanente. Proceso de Trabajo. Servicios de Salud. Indicadores de Servicios. Gestión de la Salud.

\section{Introdução}

O modelo atual da Educação Permanente em Saúde (EPS) teve origem na América Latina e decorreu da necessidade de ajustes no molde profissional contemporâneo. Antes exclusivamente voltado para as habilidades técnicas e desempenho produtivo, esse atual enfoque demanda a formação com uma proposta abrangente, que considere aspectos políticos, éticos e sociais ${ }^{(1-2)}$. Entretanto, esse novo perfil necessita de uma constante avaliação do próprio serviço da EPS, por meio da reflexão da qualidade do seu processo de trabalho. Essa ação pode ser pautada em indicadores, os quais auxiliam na compreensão de situações, tendências ou mudanças ocorridas ao longo do tempo. Desta forma, podem subsidiar melhorias para o serviço ${ }^{(3)}$.

Os indicadores podem ser considerados como medidas quantitativas utilizadas para reavaliar, replanejar e reorganizar as atividades do serviço, oferecendo subsídios para a tomada de decisão, de modo que auxiliem na melhoria da qualidade do serviço prestado. Podem contribuir na compreensão de fenômenos complexos, tornando-os quantificáveis, de maneira a analisar se processo de trabalho e objetivos propostos estão sendo alcançados ${ }^{(4)}$.

Por outro lado, sua análise pode refletir nos aspectos qualitativos, já que a interpretação pode conduzir ao melhor gerenciamento, eficiência e qualidade da EPS enquanto serviço. Avedis Donabedian trouxe contribuições inerentes a essa avaliação da qualidade nas instituições de saúde que podem ser aplicadas à EPS. Ele procurava medir as condições estruturais, desde os parâmetros físicos e de treinamento de pessoal, por meio da aplicação da Teoria dos Sistemas na relação entre recursos, processo e resultado aplicados na construção de indicadores ${ }^{(5)}$. Considera-se que a qualidade do serviço da EPS possa ser avaliada quantitativamente por indicadores e qualitativamente pela análise das implicações desses nos pilares de eficácia, efetividade, eficiência, aceitabilidade, legitimidade e equidade.

O processo de análise desses indicadores conduz, portanto, ao julgamento de respostas para questões gerenciais no serviço de EPS, o que possibilita a implementação de ações de melhoria baseadas em seus padrões de qualidade. 
Desse modo, os processos de trabalho podem ser traçados e avaliados após sua implementação. Entretanto, ainda não há, na literatura, a apresentação ou elaboração de critérios para a construção e análise desses indicadores para o serviço da EPS. Então, quais são os indicadores aplicáveis à avaliação da qualidade do serviço da EPS?

O objetivo deste estudo é refletir sobre o uso de indicadores na avaliação da qualidade do serviço de educação permanente.

\section{Método}

Estudo descritivo, tipo análise reflexiva, à luz dos pilares da qualidade de Avedis Donabedian. Emergiu com base nas reflexões e leituras aprofundadas dos pressupostos de estrutura, processo e resultados, bem como suas implicações nos pilares da qualidade dos serviços: eficácia, efetividade, eficiência, otimização, aceitabilidade, legitimidade e equidade ${ }^{(5)}$.

Iniciou-se a estratégia de revisão integrativa, mas, pela incipiência dos estudos, chegou-se no estudo reflexivo sustentado pelo referencial teórico proposto.

\section{Resultados}

A proposição de ações da EPS deve responder à necessidade do serviço com vistas à transformação da realidade. A reflexão acerca dos pilares da qualidade permite a definição conceitual proposta por Donabedian ${ }^{(5)}$, bem como a operacional, entendida como o modo de essas ações serem medidas no serviço da EPS (Quadro 1).

Quadro 1 - Definição conceitual, segundo os pilares da qualidade propostos por Donabedian, e definição operacional adaptadas à Educação Permanente em Saúde

\begin{tabular}{|c|c|c|}
\hline $\begin{array}{l}\text { Pilar da } \\
\text { qualidade }\end{array}$ & Definição conceitual & Definição operacional \\
\hline Eficácia & $\begin{array}{l}\text { O melhor que se pode fazer nas } \\
\text { condições mais favoráveis, dado } \\
\text { o estado do paciente e mantidas } \\
\text { constantes as demais circunstâncias. }\end{array}$ & $\begin{array}{l}\text { Planejamento anual das atividades em } \\
\text { condições ideais, medido com base no } \\
\text { total anual de ações e analisado pelas } \\
\text { atividades realmente ofertadas por meio } \\
\text { da média anual de ações realizadas, } \\
\text { cancelamentos e tempo médio das } \\
\text { capacitações. }\end{array}$ \\
\hline Efetividade & $\begin{array}{l}\text { Melhoria na saúde alcançada ou } \\
\text { alcançável nas condições usuais da } \\
\text { prática cotidiana. }\end{array}$ & $\begin{array}{l}\text { Relações entre as avaliações pré e } \\
\text { pós-atividade medidas pela taxa de } \\
\text { efetividade das ações realizadas. }\end{array}$ \\
\hline Eficiência & $\begin{array}{l}\text { Medida do custo com o qual uma } \\
\text { dada melhoria na saúde é alcançada. }\end{array}$ & $\begin{array}{l}\text { Custo efetivo das ações com base no total } \\
\text { investido anual. }\end{array}$ \\
\hline Otimização & $\begin{array}{l}\text { Torna-se relevante, à medida que os } \\
\text { efeitos do cuidado da saúde não são } \\
\text { avaliados de forma absoluta, mas } \\
\text { relativamente aos custos. }\end{array}$ & $\begin{array}{l}\text { Relação entre efetividade e eficiência } \\
\text { com base na relação entre a taxa de } \\
\text { melhoramento e o custo investido. }\end{array}$ \\
\hline Aceitabilidade & $\begin{array}{l}\text { Sinônimo de adaptação do cuidado } \\
\text { aos desejos, expectativas e valores } \\
\text { dos pacientes e de suas famílias. }\end{array}$ & $\begin{array}{l}\text { Contentamento com as atividades } \\
\text { propostas com base no abandono na } \\
\text { sequência dos treinamentos e na satisfação } \\
\text { dos profissionais. }\end{array}$ \\
\hline Legitimidade & $\begin{array}{l}\text { Aceitabilidade do cuidado da forma } \\
\text { em que é vista pela comunidade ou } \\
\text { sociedade em geral. }\end{array}$ & $\begin{array}{l}\text { Receptividade dos profissionais com } \\
\text { as atividades, refletida com base na } \\
\text { frequência e média das atividades } \\
\text { propostas. }\end{array}$ \\
\hline Equidade & $\begin{array}{l}\text { Princípio pelo qual se determina } \\
\text { o que é justo ou razoável na } \\
\text { distribuição do cuidado e de seus } \\
\text { benefícios entre os membros de uma } \\
\text { população. }\end{array}$ & $\begin{array}{l}\text { Média dos treinamentos ofertados por } \\
\text { meio da proposição de treinamentos pelos } \\
\text { profissionais, pela gestão da instituição } \\
\text { e pela própria Educação Permanente em } \\
\text { Saúde. }\end{array}$ \\
\hline
\end{tabular}

Fonte: Elaboração própria. 
Essas definições possibilitaram propor-se que indicadores de monitoramento das ações da EPS sejam construídos em duas perspectivas: com base nos profissionais envolvidos nas ações e na gestão do serviço. Os indicadores para a avaliação e realização das ações que considerem aspectos referentes aos profissionais estão apresentados no Quadro 2.

Quadro 2 - Indicadores de monitoramento das ações da Educação Permanente em Saúde com base nos profissionais envolvidos nas ações e na gestão do serviço, segundo Conceito e Cálculo

\begin{tabular}{|c|c|c|}
\hline Indicador & Conceito & Cálculo \\
\hline \multirow{2}{*}{$\begin{array}{l}\text { Frequência } \\
\text { nas atividades } \\
\text { propostas }\end{array}$} & \multirow{2}{*}{$\begin{array}{l}\text { Relação entre o número dos } \\
\text { profissionais presentes na atividade } \\
\text { proposta e o total de profissionais da } \\
\text { instituição. }\end{array}$} & Número de profissionais presentes \\
\hline & & $\overline{\text { Total de profissionais da instituição }}$ \\
\hline \multirow{2}{*}{$\begin{array}{l}\text { Frequência } \\
\text { nas atividades } \\
\text { propostas } \\
\text { por categoria } \\
\text { profissional }\end{array}$} & \multirow{2}{*}{$\begin{array}{l}\text { Relação entre o número dos } \\
\text { profissionais presentes por categoria } \\
\text { profissional na atividade proposta e o } \\
\text { total de profissionais da instituição. }\end{array}$} & $\begin{array}{l}\text { Número de profissionais presentes por } \\
\text { categoria }\end{array}$ \\
\hline & & $\overline{\text { Total de profissionais da instituição }}$ \\
\hline \multirow{2}{*}{$\begin{array}{l}\text { Média das } \\
\text { atividades por } \\
\text { profissionais }\end{array}$} & \multirow{2}{*}{$\begin{array}{l}\text { Relação entre o número de } \\
\text { profissionais da instituição e o } \\
\text { número de ações propostas. }\end{array}$} & Número de profissionais na instituição \\
\hline & & $\overline{\text { Número de ações propostas }}$ \\
\hline \multirow{2}{*}{$\begin{array}{l}\text { Abandono na } \\
\text { sequência dos } \\
\text { treinamentos }\end{array}$} & \multirow[t]{2}{*}{$\begin{array}{l}\text { Taxa de profissionais que desistiram } \\
\text { de frequentar a ação. }\end{array}$} & $\begin{array}{l}\text { Número de profissionais que desistiram } \\
\text { da ação }\end{array}$ \\
\hline & & Total de profissionais da instituição \\
\hline \multirow{2}{*}{$\begin{array}{l}\text { Satisfação dos } \\
\text { profissionais } \\
\text { com a atividade }\end{array}$} & \multirow{2}{*}{$\begin{array}{l}\text { Número de profissionais satisfeitos } \\
\text { com a atividade e o total presente na } \\
\text { atividade. }\end{array}$} & Número de profissionais satisfeitos \\
\hline & & $\overline{\text { Total de profissionais presentes na ação }}$ \\
\hline
\end{tabular}

Fonte: Elaboração própria.

Esses indicadores permitem que os índices esperados e os obtidos em cada ação proposta pelo serviço da EPS possam ser analisados e considerados no planejamento de atividades $a$ posteriori. Do mesmo modo, a análise de fatores inerentes ao serviço precisa ser considerada e avaliada, conforme Quadro 3.

Quadro 3 - Indicadores do serviço da Educação Permanente em Saúde, segundo conceito e cálculo

\begin{tabular}{|c|c|c|}
\hline Indicador & Conceito & Cálculo \\
\hline $\begin{array}{l}\text { Total anual de } \\
\text { ações propostas }\end{array}$ & Soma das ações realizadas em um ano. & Ação 1+ ação $2 \ldots$ ação n \\
\hline $\begin{array}{l}\text { Média anual de } \\
\text { ações realizadas }\end{array}$ & $\begin{array}{l}\text { Relação entre o número de ações } \\
\text { realizadas e o número de ações } \\
\text { propostas. }\end{array}$ & $\frac{\text { Número de ações propostas }}{\text { Número de ações realizadas }}$ \\
\hline $\begin{array}{l}\text { Tempo médio } \\
\text { das capacitações }\end{array}$ & $\begin{array}{l}\text { Relação entre o número de horas das } \\
\text { capacitações e o número de atividades } \\
\text { realizadas. }\end{array}$ & $\frac{\text { Número de horas das capacitações }}{\text { Número de atividades realizadas }}$ \\
\hline $\begin{array}{l}\text { Efetividade das } \\
\text { ações realizadas }\end{array}$ & $\begin{array}{l}\text { Relação entre a pontuação pré e pós- } \\
\text {-teste e o número total de participantes }\end{array}$ & $\frac{\text { Pontuação obtida na avaliação }}{\text { Número de profissionais presentes }}$ \\
\hline $\begin{array}{l}\text { Cancelamento } \\
\text { das ações }\end{array}$ & $\begin{array}{l}\text { Relação entre o número de ações } \\
\text { canceladas e o total das ações } \\
\text { planejadas. }\end{array}$ & $\frac{\text { Número de ações canceladas }}{\text { Total das ações planejadas }}$ \\
\hline $\begin{array}{l}\text { Total anual de } \\
\text { valor investido }\end{array}$ & $\begin{array}{l}\text { Total do valor investido nas ações } \\
\text { anualmente. }\end{array}$ & $\frac{\text { Valor na ação } 1+\text { Valor da ação } 2 \ldots}{\text { valor da ação n }}$ \\
\hline
\end{tabular}

Fonte: Elaboração própria 
Pela análise dos indicadores gerenciais do serviço da EPS pode ser analisado o resultado obtido com base no que foi planejado. Com isso, novas estratégias podem ser idealizadas para o próximo ano de atividades, considerando o resultado anterior.
Com a aplicação do resultado dos indicadores levantados dos profissionais e do serviço, torna-se possível o seu uso como uma ferramenta de trabalho da EPS, conforme ilustra a Figura 1.

Figura 1 - Aplicação dos indicadores no processo de trabalho da Educação Permanente em Saúde

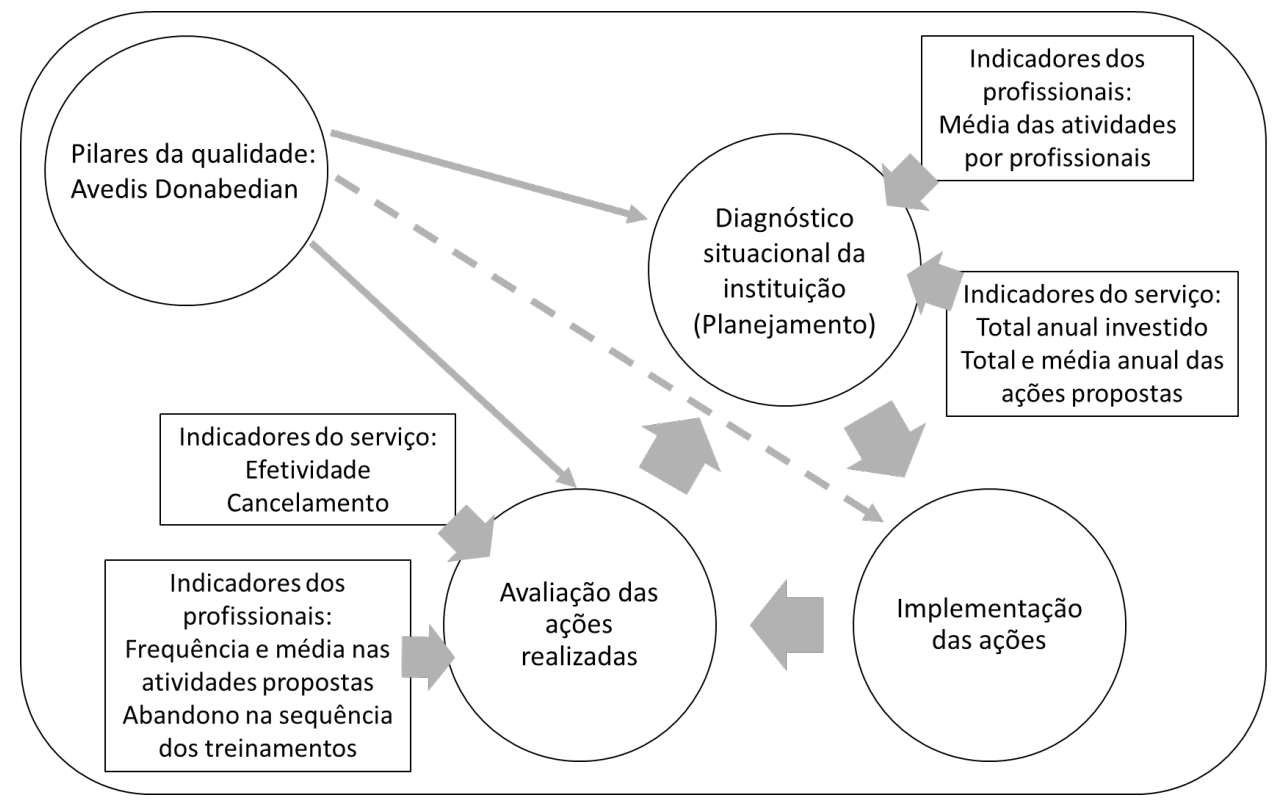

Fonte: Elaboração própria.

A construção dos indicadores à luz dos pilares da qualidade de Avedis Donabedian pode interferir no planejamento das ações com base no diagnóstico situacional. Após a realização das ações, a avaliação considera o resultado obtido, o que promove um novo diagnóstico situacional a ser utilizado em futuros planejamentos. Com isso, o processo de análise desses indicadores torna-se cíclico, de modo a planejar e avaliar com base nos indicadores pré-estabelecidos do serviço de EPS.

\section{Discussão}

Para a prática gerencial do serviço em educação permanente, faz-se necessária a reflexão dos fatores relacionados à estrutura organizacional da instituição nas políticas assistenciais, educacionais e gerenciais em saúde e do perfil dos recursos humanos. Além disso, precisam ser avaliados os programas e as propostas de trabalho, recursos financeiros e físicos disponíveis e as expectativas da clientela atendida ${ }^{(6)}$.

Essas propostas vão ocorrer fazendo-se uso da tríade estrutura (recursos humanos e materiais), processo de trabalho e resultados, por meio da qual os indicadores são elaborados, avaliados e qualificados. Vem a reflexão donabediana de ênfase nas métricas e medidas de qualidade em saúde por meio dos seus pilares ${ }^{(2)}$. Para tanto, reflexões diferenciadas dos profissionais e do próprio serviço podem levar a análises específicas dos indicadores da EPS. Daí a necessidade de formulação de indicadores separados e discussões pormenorizadas, conforme a seguir.

\section{Análise de indicadores relacionados ao profissional}

Os indicadores propostos na perspectiva dos profissionais levam em consideração a frequência e a média nas atividades realizadas, o 
abandono e a satisfação dos envolvidos. Esses indicadores podem interferir nos pilares de aceitabilidade e legitimidade ${ }^{(7)}$.

A aceitabilidade traduz-se na existência de temas pautados em consonância com os desejos dos usuários e do cotidiano de trabalho e podem ser motivações para realização das práticas educativas. Isso quer dizer que, se somente demandas da instituição e da EPS apontarem para quais atividades educativas precisam ser realizadas, a satisfação dos profissionais pode ser comprometida. Com base nisso, vem a maior necessidade de diálogo com as demandas apontadas pelos profissionais, bem como estratégias de divulgação mais assertivas, visando aumentar a efetividade e a aceitabilidade das ações ${ }^{(8)}$.

Do mesmo modo, a falta de motivação com as atividades propostas precisa ser mensurada pelo abandono na sequência dos treinamentos. Isso conduz à necessidade de estimular os profissionais no processo educativo e na responsabilidade com a própria qualificação e capacitação. Nesse sentido, o aumento da carga de trabalho dos profissionais pode trazer o redesenho do trabalho e repercutir na descontinuidade das atividades propostas. Estudos apontam ainda dificuldades quanto à realização de ações da EPS nas instituições de saúde, principalmente em razão da baixa adesão dos profissionais e da resistência na apreensão de novos conhecimentos ${ }^{(9)}$.

Esses indicadores relacionados ao profissional trazem o pensamento de articulação das estratégias com vistas à inclusão do processo educativo no cotidiano do trabalho. Um conjunto de ações pode ser articulado por uma relação clara entre os profissionais que constituem uma instituição de saúde, o serviço da EPS e a gestão institucional, tanto no sentido de flexibilizar os horários das atividades quanto no tocante ao seu planejamento. Essas intervenções podem aumentar as taxas de adesão e melhorar o resultado das ações ${ }^{(10)}$.

As ações educativas precisam relacionar-se à realidade das unidades assistenciais, para que ocorram no contexto do trabalho e tenham disponibilidade de horários adequados às escalas dos profissionais. Para tal, há necessidade de apoio institucional, questão que perpassa pela adequação do ambiente físico e de recursos humanos, mas também pela necessidade de planejamento, coparticipação, comprometimento e disponibilidade das chefias e equipes de trabalho do serviço da EPS.

Além disso, a legitimidade é um pilar determinante no processo reflexivo em EPS, de modo que interfere na relação entre os profissionais e o processo educativo prestado. Desta forma, como a EPS é vista pelos profissionais precisa ser incluída na avaliação. Na tentativa de medir a qualidade, pode ser frágil considerar os fatores de avaliação formativa individualizada, mas o dado numérico em sua amplitude pode auxiliar na avaliação geral e na proposição de alterações de novas atividades com a mesma temática ${ }^{(11)}$.

\section{Indicadores relacionados ao serviço de Educação Permanente em Saúde}

Os indicadores de gestão do serviço da EPS englobam o total de atividades realizadas, a média anual de ações e o tempo médio de cada capacitação. Deste modo, permitem a avaliação global da oferta de atividades da EPS com base na análise de sua eficácia, efetividade, eficiência, otimização e equidade. Apesar de não avaliarem a qualidade e a efetividade da ação educativa proposta, possibilitam dimensionar o planejamento de ações futuras, considerando os recursos disponíveis, bem como a análise das atividades ofertadas.

Nesse sentido, os indicadores do serviço da EPS emergem, principalmente, da capacidade de planejamento das atividades. Isso engloba o planejamento de custo, a organização do trabalho, projeto das ações já associados às dificuldades locais, comunicações e à tomada de decisões, contribuindo no aperfeiçoamento das estruturas e no processo de trabalho institucional ${ }^{(12)}$. Desta forma, o total anual de ações propostas e do valor investido depende de elaboração de um plano de atividades prévio e que considere as possibilidades da instituição.

Em contrapartida, a análise da sua efetividade considera o alcance da ação naquele(s) determinado(s) grupo(s) de profissional(is) após as realizações das atividades. Um dos modos de fazer 
essa avaliação é pela aplicação de questionários pré e pós-testes, que medem comparativamente o coeficiente de acerto dos participantes. Avaliar o conhecimento de base com o apoio de pré-testes pode auxiliar na avaliação das alterações do conhecimento e na compreensão do real alcance da atividade ${ }^{(13)}$. Esse conhecimento avaliado pode ser uma estratégia de mudança de comportamento e, apesar de não poder ser utilizado de modo isolado, tem potencial de servir de base no conhecimento adquirido na ação.

O cancelamento das atividades é outro indicador de interesse da EPS. O planejamento depende de diversos fatores internos ou externos ao serviço que interferem diretamente na efetiva realização das ações. Desta forma, as atividades dependem de recursos humanos e materiais que precisam ser analisados previamente, bem como do preparo prévio das estratégias adaptadas aos diferentes estilos de aprendizagem, às necessidades dos participantes e à estrutura oferecida pela instituição. Caso um desses aspectos entre em desalinho, pode ocorrer o cancelamento da atividade e o motivo precisa ser analisado e considerado pelo serviço no planejamento futuro ${ }^{(14)}$.

Nesse sentido, esse indicador pode interferir diretamente no quantitativo das ações, medido pela média anual de ações realizadas e pelo tempo médio das capacitações e, consequentemente, na sua eficácia. Torna-se importante esta análise por permitir o comparativo ao longo do tempo e perceber os avanços do serviço. A oferta de atividades por procedimentos ou por categorias profissionais requer análise da necessidade de se resguardar o caráter multiprofissional e interdisciplinar da EPS e também precisa ser avaliada continuamente. Acrescenta-se ainda a necessidade de aprendizado no tempo de prática cotidiana do trabalho, a fim de possibilitar maior articulação entre o ensino e a prática realizada ${ }^{(15)}$.

Sobre o indicador total anual de valor investido, o financiamento é um fator que interfere diretamente na eficiência do serviço da EPS. Isso porque possibilita a autonomia das ações e evita a verticalização, bem como traz a possibilidade de adequação das propostas com as necessidades do serviço ${ }^{(8)}$. Por isso, o perfil do investimento precisa ser analisado continuamente, com vistas à avaliação da quantia investida relacionada ao perfil institucional e às necessidades do serviço.

A análise individual desses indicadores pode apontar informações de interesse no serviço da EPS. Entretanto, a relação entre eles pode conduzir à análise de um perfil importante e associar causa e efeito de necessidade de mudanças no serviço. A redução de treinamentos, seja pelo total anual, tempo médio ou taxa de cancelamentos, pode ter relação direta com o financiamento. A baixa satisfação dos participantes pode ter relação com a frequência nos treinamentos e com o abandono na sequência dos treinamentos. Por isso, a adoção de mais de um indicador pode ser importante e auxiliar na avaliação e no planejamento das ações no serviço.

\section{Avaliação dos indicadores de qualidade do serviço da Educação Permanente em Saúde}

A qualidade da EPS pode interferir em elementos associados à sua prática gerencial enquanto serviço. A análise dos seus indicadores deve implicar em: eficácia, ao alcançar as metas propostas; eficiência, a fim de utilizar o uso racional dos recursos; efetividade na análise dos seus resultados sociais; equidade, quando determina o que é justo ou razoável na distribuição dos recursos e benefícios; e satisfação, ao associar expectativas e contentamento dos profissionais $^{(5,16)}$.

Ao agrupar informações de interesse, a consolidação dos aspectos da realidade institucional e dos profissionais, por meio dos indicadores, pode aperfeiçoar a gestão da EPS e auxiliar no desenvolvimento de políticas internas. Além disso, torna-se possível a comparação entre as informações antes e depois dos resultados obtidos com a atividade, bem como subsidiar a avaliação das ações que justifiquem a previsão e a provisão de propostas, o que implica na análise da qualidade do serviço da $\operatorname{EPS}^{(17-18)}$. Desta forma, essa análise tem a função de verificar o aperfeiçoamento das ações, assim como a necessidade de (re)orientação e a (re)condução dos processos. Por meio dos indicadores propostos, tanto os profissionais quanto o serviço são fontes de informações importantes para o serviço da EPS, de modo que 
refletem as características das práticas institucionais e cotidianas.

Por outro lado, a despeito das contribuições na construção e na avaliação dos indicadores, os pressupostos donabedianos podem precisar de outras reflexões na sustentação da EPS. O reflexo na qualidade do serviço da instituição de saúde e na melhoria da assistência são de avaliação necessária, mas transcendem a possibilidade de avaliação individualizada do serviço da EPS.

A reflexão sobre o impacto dos treinamentos pode precisar da articulação de outros setores, como da Comissão de Controle de Infecção Hospitalar (CCIH), Serviço de Saúde Ocupacional do Trabalhador, Setor de Qualidade, dentre outros. Por isso, pode-se refletir sobre a amplitude da EPS com base na melhora de indicadores de outros serviços, o que contribui ainda mais para as implicações das ações da EPS. Um treinamento, por exemplo, de lavagem das mãos pode interferir diretamente na redução de infecções hospitalares e precisa de discussões em conjunto com a CCIH, tanto no levantamento da necessidade quanto na sua avaliação posterior.

Por fim, este estudo limita-se a refletir sobre as contribuições dos pilares da qualidade donabedianos da EPS na construção e avaliação dos seus indicadores. Entretanto, entende-se a larga amplitude de ações do serviço e sua interferência nos demais setores de saúde e ratifica-se como a reflexão de suas práticas precisa ser considerada na qualificação dos profissionais e na melhoria da assistência prestada.

\section{Conclusão}

As reflexões sobre o uso de indicadores na avaliação da qualidade do serviço de educação permanente foram possíveis e podem contribuir com o diagnóstico situacional no planejamento das ações e na avaliação dessas por meio da análise da qualidade na eficácia, eficiência, efetividade, equidade e satisfação. Desta forma, auxiliam na compreensão das potencialidades e fragilidades na condução das atividades e podem propiciar melhoramentos no planejamento e na organização futuros. Para tanto, considera as implicações para os profissionais e para o próprio serviço da EPS. Entretanto, novos estudos quantitativos e qualitativos podem ser necessários com vistas à validação e aplicabilidade desses indicadores, de modo a contribuir com a visibilidade da EPS e auxiliar na análise de suas demandas.

\section{Colaborações:}

1 - concepção, projeto, análise e interpretação dos dados: Graziele Ribeiro Bitencourt;

2 - redação do artigo e revisão crítica relevante do conteúdo intelectual: Graziele Ribeiro Bitencourt, Andreia Fabia de Melo Ferreira, Maria Helena de Souza Praça do Amaral, Stella Maris Gomes Renault, Jaqueline Olímpio da Silva e Katerine Moraes dos Santos;

3 - aprovação final da versão a ser publicada: Graziele Ribeiro Bitencourt, Andreia Fabia de Melo Ferreira, Maria Helena de Souza Praça do Amaral, Stella Maris Gomes Renault, Jaqueline Olímpio da Silva e Katerine Moraes dos Santos.

\section{Referências}

1. Lemos CLS. Educação permanente em saúde no Brasil: educação ou gerenciamento permanente? Ciênc saúde coletiva. 2016;21(3):913-22. DOI: 10.1590/1413-81232015213.08182015

2. Ayanian JZ, Markel H. Donabedian's lasting framework for health care quality. N Engl J Med. 2016;375(3):205-7. DOI:10.1056/nejmp1605101

3. Cardoso MLM, Costa PP, Costa DM, Xavier C, Souza RMP. A política nacional de educação permanente em saúde nas escolas de saúde pública: reflexões a partir da prática. Ciênc saúde coletiva. 2017;22(5):1489-500. DOI: 10.1590/1413-81232017225.33222016

4. Cavalcante OS, Rossaneis MA, Haddad MCL, Gabriel CS. Indicadores de qualidade utilizados no gerenciamento da assistência de enfermagem hospitalar. Rev enferm UERJ. 2015;23(6):787-93. DOI: $10.12957 /$ reuerj.2015.7052

5. Bitencourt GR, Santana RF, Menezes AK, Cimador F, Delvalle R. Fundamentos filosóficos e conceitos da classificação de resultados: contribuições na avaliação de enfermagem. Rev enferm UFPE. 2016;10(5):787-93. DOI: 10.5205/ reuol.9284-81146-1-SM.1005sup20162 
6. Brasil. Ministério da Saúde. Secretaria de Gestão do Trabalho e da Educação na Saúde. Departamento de Gestão da Educação na Saúde. Política de educação e desenvolvimento para o SUS: caminhos para a educação permanente em saúde, polos de educação permanente em saúde [Internet]. Brasília (DF); 2004. (O SUS de A a Z) [cited 2020 May 5]. Available from: http://nephrp. com.br/site/wp-content/uploads/2017/03/02Pol\%C3\%ADtica-de-Educa\%C3\%A7\%C3\%A3o-eDesenvolvimento-para-o-SUS-Caminhos-paraa-Educa\%C3\%A7\%C3\%A3o-Permanente-emSa\%C3\%BAde.pdf

7. Hu R, Liao Y, Du Z, Hao Y, Liang H, Shi L. Types of health care facilities and the quality of primary care: a study of characteristics and experiences of chinese patients in Guangdong Province, China. BMC Health Serv Res. 2016;16(1):335. DOI: 10.1186/s12913-016-1604-2

8. Sena RR, Grillo MJC, Pereira L, Belga SMMF, França BD, Freitas CP. EPS nos serviços de saúde: atividades educativas desenvolvidas no estado de Minas Gerais, Brasil. Rev Gaúcha Enferm. 2017;38(2):e64031. DOI: $10.1590 / 1983-1447.2017 .02 .64031$

9. Sade PMC, Peres AM, Brusamarelo T, Mercês NNA, Wolff LDG, Lowen IMV. Demandas da EPS de enfermagem em um hospital de ensino. Cogitare enferm. 2019;24:e57130. DOI: 10.5380/ ce.v24i0.57130

10. Lavich CRP. Ações de educação permanente dos enfermeiros facilitadores de um núcleo de educação em enfermagem. Rev Gaúcha Enferm. 2017;38(1):e62261. DOI: 10.1590/1983-1447.2017.01.62261

11. Bartlett M, McKinley JPB. Do quality indicators for general practice teaching practices predict good outcomes for students? Educ Prim Care. 2016;27(4):271-9. DOI: 10.1080/14739879.2016.1175913
12. Folb BL, Klem ML, Youk AO. Continuing education for systematic reviews: a prospective longitudinal assessment of a workshop for librarians. J Med Libr Assoc. 2020;108(1):36-46. DOI: 10.5195/ jmla.2020.492

13. Ganassin GS, Silva EM, Pimenta AP, Marcon SS. Efetividade da intervenção educativa no conhecimento de homens relacionado às doenças cardiovasculares. Acta Paul Enferm. 2016;29(1):38-46. DOI: 10.1590/1982-0194201600 006

14. Campos KFC, Marques RC, Silva KL. EPS: discursos dos profissionais de uma unidade básica de saúde. Esc Anna Nery. 2018;22(4):e20180172. DOI: 10. 1590/2177-9465-ean-2018-0172

15. Breytenbach C, Ham-Baloyi T, Jordan PJ. An integrative literature review of evidence-based teaching strategies for nurse educators. Nurs Educ Perspect. 2017;38(4):193-7. DOI: 10.1097/01. nep.0000000000000181

16. Ferreira L, Barbosa JSA, Esposti CDD, Cruz MM. Educação permanente em saúde na atenção primária: uma revisão integrativa da literatura. Saúde debate. 2019;43(120):223-39. DOI: 10. 1590/0103-1104201912017

17. Costa MAR, Souza VS, Teston EF, Spigolon DN, Matsuda LM. Educação permanente em saúde: a concepção freireana como subsídio à gestão do cuidado. Rev Fund Care Online. 2018;10(2):558-64. DOI: $10.9789 / 2175-5361 . r p c f o . v 10.6368$

18. Wellings CA1, Gendek MA, Gallagher SE. Evaluating continuing nursing education: a qualitative study of intention to change practice and perceived barriers to knowledge translation. J Nurses Prof Dev. 2017;33(6):281-6. DOI: 10.1097/ NND.0000000000000395

Recebido: 15 de maio de 2020

Aprovado: 20 de julho de 2020

Publicado: 25 de novembro de 2020

A Revista Baiana de Enfermagem utiliza a Licença Creative Commons - Atribuição-NãoComercial 4.0 Internacional. https://creativecommons.org/licenses/by-nc/4.0/ Este artigo é de acesso aberto distribuído sob os termos da Licença Creative Commons (CC BY-NC). Esta licença permite que outros remixem, adaptem e criem a partir do seu trabalho para fins não comerciais. Embora os novos trabalhos tenham de lhe atribuir o devido crédito e não possam ser usados para fins comerciais, os usuários não têm de licenciar esses trabalhos derivados sob os mesmos termos. 